\title{
Insight into k13-propeller gene polymorphism and ex vivo DHA-response profiles from Cameroonian isolates
}

\author{
Sandie Menard', Joëlle Njila Tchoufack², Christelle Ngou Maffo², Sandrine E. Nsango 2,3, Xavier Iriart1,4, \\ Luc Abate ${ }^{5}$, Majoline Tchioffo Tsapi ${ }^{5}$, Parfait H. Awono-Ambéné2 ${ }^{2}$ Francis A. Abega Mekongo ${ }^{6}$, Isabelle Morlais ${ }^{5 \dagger}$ \\ and Antoine Berry ${ }^{1,4^{*}+}$
}

\begin{abstract}
Background: The spread of Plasmodium falciparum resistance to artemisinin derivatives in Southeast Asia is a major source of concern and the emergence of resistance in Africa would have dramatic consequences, by increasing malaria mortality and morbidity. It is therefore urgent to implement regular monitoring in sentinel sites in sub-Saharan Africa using robust and easy-to-implement tools. The prevalence of k13-propeller mutations and the phenotypic profiles are poorly known in sub-Saharan Africa. Here, the k13-propeller polymorphism was compared to both ex vivo susceptibility to DHA and early parasitological and clinical responses to artemisinin combination therapy (ACT).

Methods: Plasmodium falciparum isolates were collected in 2015 in Yaoundé (Cameroon) from patients treated with dihydroartemisinin-piperaquine combination. Samples were analysed for their susceptibility to artemisinin using the k13-propeller sequencing, the ex vivo ring-stage survival assay, the in vivo parasite positive rate and the clinical statute at day 2.

Results: None of the collected isolates revealed the presence of resistance mutations in the k13-propeller sequence. The median ring-stage survival rate for all the 64 interpretable isolates after a 6 -hour pulse of $700 \mathrm{nM}$ dihydroartemisinin was low, $0.49 \%$ (IQR: 0-1.3). Total parasite clearance was observed for $87.5 \%$ of patients and the remaining parasitaemic isolates (12.5\%) showed a high reduction of parasite load, ranging from 97.5 to $99.9 \%$. Clinical symptoms disappeared in $92.8 \%$ of cases.

Conclusion: This study demonstrated the absence of k13-resistant genotypes in P. falciparum isolates from Cameroon. Only synonymous mutations were found with a low prevalence (4.3\%). A good association between $\mathrm{k} 13$ genotypes and the ex vivo ring-stage survival assay or parasitological and clinical data was obtained. These results give a baseline for the long-term monitoring of artemisinin derivative efficacy in Africa.
\end{abstract}

Keywords: Artemisinins, Dihydroartemisinin, Resistance, Plasmodium falciparum, Ring-stage survival assay, K13, Clearance, Cameroon

\section{Background}

Malaria remains a major public health problem largely due to Plasmodium falciparum drug resistance. Artemisinin (ART)-based combination therapy (ACT) is the

\footnotetext{
*Correspondence: berry.a@chu-toulouse.fr

†Isabelle Morlais and Antoine Berry are senior authors contributed equally to this work

${ }^{1}$ Centre de Physiopathologie de Toulouse Purpan, INSERM U1043, CNRS

UMR5282, Université de Toulouse, Toulouse, France

Full list of author information is available at the end of the article
}

first-line treatment for falciparum malaria, used worldwide to ensure the highest cure rates and to reduce the spread of drug resistance [1]. Unfortunately ART-resistant $P$. falciparum was reported on the Cambodia/Thailand border in 2008, characterized by a reduced parasite clearance in patients as primary parasitological feature [2]. ART-resistance has spread and today the resistance has been confirmed in five countries of the Greater Mekong Sub-region [3-7]. The identification of ART resistance in Asia is reminiscent of previously emerged 
resistance to chloroquine and sulfadoxine-pyrimethamine, which appeared in the same region and rather quickly reached Africa with dramatic consequences of increased malaria mortality and morbidity $[8,9]$. The only question for Africa is to know when and where resistance to artemisinins will appear. It is, therefore, urgent to implement regular monitoring in several sentinel sites in sub-Saharan Africa using robust and easy-toimplement tools.

In the presence of ART derivatives, resistant parasites are able to stop their life cycle in ring stage and enter in quiescence [10-12]. Because of this particular mechanism of resistance, different from other anti-malarials, the standard determination of $50 \%$ inhibitory concentration (IC50) is not informative for assessing the level of resistance of $P$. falciparum isolates to ART derivatives. Today, in addition to clinical trials, two new tools are available for surveillance of ART resistance: (i) the detection of single nucleotide polymorphisms (SNPs) in the PF3D7_1343700 kelch propeller ( $k 13$-propeller domain) that have been identified as a key causal determinant in Southeast Asia [13, 14]; and, (ii) the ring-stage survival assay (RSA) [15] that gives phenotypic information, evaluating by microscopic counting the proportion of viable parasites after a 6-hour exposure to $700 \mathrm{nM}$ dihydroartemisinin (DHA).

A total of 186 different $k 13$ alleles have been reported [16] in Southeast Asia so far. In Africa, different nonsynonymous $k 13$-propeller-region mutations have been reported but remain rare and highly diverse [17-19]. Moreover, these SNPs were different from those in Asia and have never been associated with increased parasite half-life values or clinical failures [19-22]. So the monitoring of ART susceptibility must be implemented in Africa but it will require the establishment of a baseline correlation between genotypic and phenotypic data. Furthermore, to date only one study performed in Uganda evaluated the ex vivo RSA associated with $k 13$ polymorphism on few isolates $(n=43)$, and this study did not report any increased survival rate associated with nonsynonymous $k 13$ mutation [17].

In this work, the polymorphism in the k13-propeller domain of $P$. falciparum isolates from Yaoundé, Cameroon was evaluated and these genotypic data were associated to the in vivo and ex vivo phenotypic results. Thus, all these results provided baseline data of survival rate profiles according to the $k 13$ gene polymorphism of African P. falciparum isolates.

\section{Methods}

\section{Study site and design}

A prospective study was carried out between March and June 2015 in the Nkol-Eton healthcare centre in Yaoundé intra-muros, Cameroon. A finger-prick, Giemsa-stained, thick smear was performed on all patients with symptoms of malaria to assess the presence of $P$. falciparum asexual parasites. Patients $\geq 4$ years old, with uncomplicated falciparum malaria and $>1000$ asexual parasites per $\mu \mathrm{l}$ on positive thick blood smear, were enrolled as volunteers after they or their parents had signed an informed consent form. Patients who had any anti-malarial treatment within the 15-day period before falciparum diagnosis were excluded from the study. Patients with severe malaria (defined by WHO criteria) or with vomiting preventing any oral treatment were referred to the hospital for adequate care and were not included in the study. At enrolment (day 0), study subjects' parasite density, body temperature and body weight were recorded along with any other symptoms and signs.

The patients enrolled in the study were given a threeday course of DHA $40 \mathrm{mg} /$ piperaquine (PPQ) $320 \mathrm{mg}$ (Malacur $^{\circledR}$, Elder Pharmaceuticals LTD, India) according to recommendations. The prescription was based on body weight ( 10 to $<20 \mathrm{~kg}$ : $40 \mathrm{mg}$ DHA + $320 \mathrm{mg}$ PPQ or one tablet; 20 to <40 kg: $80 \mathrm{mg}$ DHA $+640 \mathrm{mg}$ PPQ or two tablets; $\geq 40 \mathrm{~kg}: 120 \mathrm{mg}$ DHA + $960 \mathrm{mg}$ PPQ or three tablets). To ensure good treatment compliance the patients took the first dose of DHA/PPQ in Nkol-Eton healthcare centre in presence of a nurse and went home with the second dose. Twenty-four hours after enrolment, a phone call to patients ensured that the second dose was well taken. The last third dose of DHA/PPQ was given during the follow-up visit on day 2 at the Nkol-Eton healthcare centre in presence of a nurse. In case of treatment failure, a standard replacement anti-malarial therapy (quinine salts) was administered according to $\mathrm{WHO}$ recommendations.

\section{Ethics statement}

All procedures involving human subjects used in this study were approved by the Cameroonian National Ethical Committee (statement $n^{\circ}: 2015 / 04 / 582 / C E / C N E R S H /$ $\mathrm{SP})$.

\section{Ex-vivo ring-stage survival assay (RSA)}

Venous blood samples were collected into acid-citratedextrose $(\mathrm{ACD})$ vacutainers at day 0 before treatment, and were kept at $4{ }^{\circ} \mathrm{C}$ until processing. The ex vivo RSA was performed directly from the ACD blood sample within $24 \mathrm{~h}$ after blood collection, as previously described [15]. Briefly, after elimination of plasma, white cells and anticoagulant, $P$. falciparum parasites with a parasitaemia between 0.1 and $1 \%$ were exposed to either $700 \mathrm{nM}$ DHA or $0.1 \%$ dimethyl sulfoxide (DMSO) (DHA solvent) for 6 hours, washed and then cultivated for $66 \mathrm{~h}$ at $37^{\circ} \mathrm{C}$ under humid, oxygen-deficient atmosphere (candle jar). Microscopic quantification of the proportion of viable parasites was performed by expert microscopists on Giemsa-stained 
thin smears to calculate the growth rate as the ratio of parasitaemia between DMSO-exposed and initial conditions, and the survival rate as the ratio of parasitaemia between DHA-exposed and DMSO-exposed conditions. Survival data were considered interpretable only if the growth rate was $\geq 1$. Equipment necessary for implementation of RSA included laminar flow hood, candle jar (without gas), $-20{ }^{\circ} \mathrm{C}$ freezer, $4{ }^{\circ} \mathrm{C}$ fridge, and cold chain.

\section{Sequencing of $k 13$-propeller domain}

DNA was extracted from venous blood samples obtained at patient enrolment (day 0) using the spin protocol of QIAamp Mini kit ${ }^{\circledR}$ (Qiagen, Hilden, Germany) according to manufacturer's recommendations. PCR amplification and sequencing of k13-propeller domain were performed, as previously described [13]. After control on $1.5 \%$ agarose gel electrophoresis, the PCR products were sequenced by ABI 3130xl Genetic Analyzer. Sequences were compared to 3D7 reference strain sequence with BioEdit Sequence Alignment Editor (version 7.2.3).

\section{Early clinical and parasitological responses to treatment}

Patients were asked to return to the healthcare centre for follow-up visits on day 2 after treatment. Each visit included completion of a standardized history form, a physical/clinical examination for recording any signs (temperature) or symptoms (headache, vomiting, diarrhoea, diffuse pain), and a finger-prick blood sample for thick smear. Blood smear slides were stained with a $10 \%$ Giemsa solution and examined microscopically under oil-immersion at $1000 \times$. Parasitaemia was determined by counting the number of asexual parasites against 500 white blood cells and the density estimated considering 8000 white blood cells per $\mu$ l. Slides at day 2 were assessed independently by two expert microscopists. A third microscopist validated any case of discordance.

Clinical and parasitological responses to treatments were classified as follows: (1) parasite negativity rates (PNRs) for the proportion of patients without any parasitaemia on day 2; (2) parasite positivity rates (PPRs) for the proportion of patients remaining parasitaemic on day 2; and, (3) early treatment failure (ETF) for patients with danger signs, complicated malaria or presence of parasitaemia on day 2 with fever (rectal temperature $\geq 38^{\circ} \mathrm{C}$ ) or parasitaemia on day 2 higher than on day 0 . Patients were excluded from the clinical and parasitological assessment if they were lost to follow-up.

\section{Statistical analysis}

All statistical tests were performed using GraphPad Prism software version 5 (GraphPad Inc., San Diego, CA, USA). Data that were not normally distributed were displayed as median along with interquartile ranges and were compared with Mann-Whitney $U$ test for two group comparisons. The Gaussian data were reported as the mean \pm standard deviation and were analysed using the $t$ test for two group comparisons. Proportions were compared using the $\chi^{2}$ test or the Fisher's exact test, as appropriate. Correlations were determined using the Spearman test. A comparison was considered statistically significant if the $\mathrm{p}$ value was $\leq 0.05$.

\section{Results}

\section{Baseline characteristics of eligible patients through the study}

Between March and June 2015, a total of 166 patients with falciparum malaria were enrolled. The flow of patients through the study is displayed in Fig. 1. The baseline characteristics of these patients are given in Table 1. The weight adjusted drug dose $(\mathrm{mg} / \mathrm{kg})$ was calculated in $98 \%$ of the patients as the body weight was available in 96 of the 98 patients. Some $76.0 \%$ of the patients $(73 / 96)$ received a total dose of DHA and PPQ components, which followed WHO recommendations (DHA: $2-10 \mathrm{mg} / \mathrm{kg}$; PPQ: $16-27 \mathrm{mg} / \mathrm{kg})$. The $24.0 \%(23 / 96)$ other patients who received a dose of DHA and PPQ components under the WHO recommendations were all over 15 years old.

\section{K13-propeller polymorphisms}

The $k 13$ gene was genotyped from 98 P. falciparum isolates out of the 166 eligible patients. For 57 patients, no blood sample was obtained because of blood collection

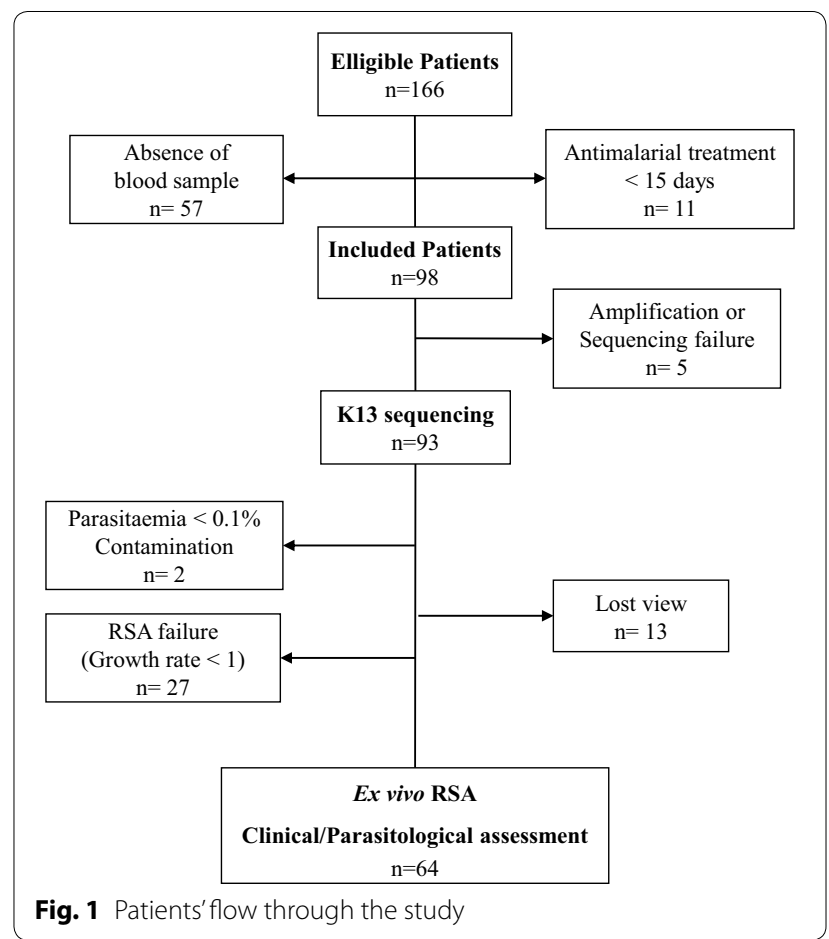


Table 1 Baseline characteristics of the patients included in the study at day 0

\begin{tabular}{|c|c|}
\hline Baseline characteristics & \\
\hline Patients (n) & 98 \\
\hline \multicolumn{2}{|l|}{ Gender } \\
\hline Sex ratio n (\%) & 0.96 \\
\hline Male & $48(49.0 \%)$ \\
\hline Female & $50(51.0 \%)$ \\
\hline \multicolumn{2}{|l|}{ Age } \\
\hline Median age (IQR) (years) & $11.5(6-18.8)$ \\
\hline$<8$ years $\mathrm{n}(\%)$ & 39 (39.8\%) \\
\hline $8-15$ years $n(\%)$ & $23(23.5 \%)$ \\
\hline$>15$ years $n(\%)$ & $36(36.7 \%)$ \\
\hline \multicolumn{2}{|l|}{ Body weight $(n=96)$} \\
\hline Median body weight (IQR) (kg) & $33(21-57)$ \\
\hline$<25$ kg n (\%) & $37(38.5 \%)$ \\
\hline$\geq 25$ kg n (\%) & $59(61.5 \%)$ \\
\hline \multicolumn{2}{|l|}{ Temperature } \\
\hline Median $(\mathrm{QQR})\left({ }^{\circ} \mathrm{C}\right)$ & $38.2(37-39.5)$ \\
\hline Fever (temperature $\geq 38^{\circ} \mathrm{C}$ ) n (\%) & $51(52.0 \%)$ \\
\hline \multicolumn{2}{|l|}{ Symptoms n (\%) } \\
\hline Headache & $66(67.3 \%)$ \\
\hline Vomiting & $42(42.9 \%)$ \\
\hline Diarrhoea & $8(8.2 \%)$ \\
\hline Diffuse pain & $9(9.2 \%)$ \\
\hline \multicolumn{2}{|l|}{ P. falciparum asexual parasite density } \\
\hline Median parasitaemia (IQR) (parasites/ $\mu \mathrm{l})$ & $42,332(17,893-87,302)$ \\
\hline \multicolumn{2}{|l|}{ Anti-malarial dose $(\mathrm{mg} / \mathrm{kg})(\mathrm{n}=96)$} \\
\hline DHA Median (IQR) & $2.5(2.0-3.0)$ \\
\hline PPQ Median (IQR) & $19.8(16.3-24)$ \\
\hline
\end{tabular}

difficulties or technical understaffing and 11 other patients used anti-malarial treatment within the 15 dayperiod before enrolment (Fig. 1). Five samples failed to amplify or did not generate high-quality sequences for analysis (Fig. 1). The $k 13$-propeller sequence polymorphism analysis of the 93 isolates revealed only wild-type profiles (95\% CI: 96.31-100\%) with four (4.3\%) synonymous changes.

\section{Ex-vivo ring-stage survival assay (RSA)}

DHA susceptibility data obtained for the $93 k 13$-sequenced samples were analysed using the ex vivo RSA (Fig. 1). The 64 isolates with an interpretable RSA (growth rate $\geq 1$ ) had a median survival rate of $0.49 \%$ (IQR: 0-1.3) (Fig. 2). Forty of the 64 (62.5\%) DHA-exposed isolates demonstrated viable parasites with a median parasitaemia of $0.012 \%$ (IQR: $0.007-0.029$ ) and a median survival rate of $1.14 \%$ (IQR: 0.6$2.2)$ whereas $24(37.5 \%)$ cultures contained only pyknotic/ dead forms (Fig. 2a). The dispersion of ex vivo RSA data was high: four isolates (6.3\%) had a survival rate higher than the mean survival rate plus 2 standard deviations (3.4\%). There were no significant differences in the growth rate $($ mean $=1.878 \pm 0.435$ vs $2.317 \pm 0.2413 ;$ t-test; $\mathrm{P}=0.644)$ or in the initial parasitaemia (mean $=0.630 \pm 0.065$ vs $0.669 \pm 0.052$; $t$-test; $\mathrm{P}=0.848$ ) between parasites with a high or a low survival rate.

\section{Early clinical and parasitological responses to treatment}

Clinical and parasitological characteristics of the studied population are shown in Table 2. The parasite clearance rate for this study was rapid as the PPR decreased to $12.5 \%$ $(8 / 64)$ on day 2 . The parasitaemia at day 2 in the PPR group was low with a median of 293 (IQR: 145-736) and a maximum of 1143 parasites per $\mu \mathrm{l}$ of blood. No patients presented ETF. Whereas some isolates remained parasitaemic at day 2, the rate of parasite decrease was high and varied between 97.5 and $99.9 \%$. The PPR at day 2 was not affected by the baseline parasite density at day 0 . Most patients (92.8\%) were totally asymptomatic within $48 \mathrm{~h}$ after drug administration. No difference in age, weight, fever, DHA/ PPQ anti-malarial doses or symptoms was observed between patients in day-2 PPR and PNR groups. No difference in survival rate in the ex vivo RSA was observed between the two groups, day 2 PPR and PNR (Fig. 2b).

\section{Discussion}

The spread of resistance threatens the global control of falciparum malaria, especially if resistance reaches subSaharan Africa, the most malaria affected area, and this makes crucial a closely monitoring of efficacy of ACT.

Here, a genotypic ( $k 13$ polymorphism) characterization of Cameroonian P. falciparum isolates was reported. Ex-vivo and in-vivo phenotypic profiles of these parasites were given for comparison in order to have a baseline picture of artemisinin susceptibility. The sequencing of the $k 13$-propeller domain from samples from Cameroon did not reveal resistance genotypes and only detected synonymous mutations at a low prevalence (4.3\%). The genotypic profile observed in this study was consistent with other African data that have not reported Asian profiles but only non-synonymous SNPs not associated with ART resistance $[16,19]$.

The ex vivo RSA performed in this study has been developed and validated in Southeast Asia to distinguish fast-clearance parasites from slow-clearance parasites in the field [15], and resistance phenotypes obtained in RSA strongly correlate with the presence of mutations in the k13-propeller domain [13]. The phenotypic data obtained from this study were in agreement with the genotypic ones. The absence of $k 13$ polymorphisms was associated with a low median of survival rate for the tested isolates $(0.49 \%)$. The high dispersion of ex vivo RSA data observed in this study could not be explained 


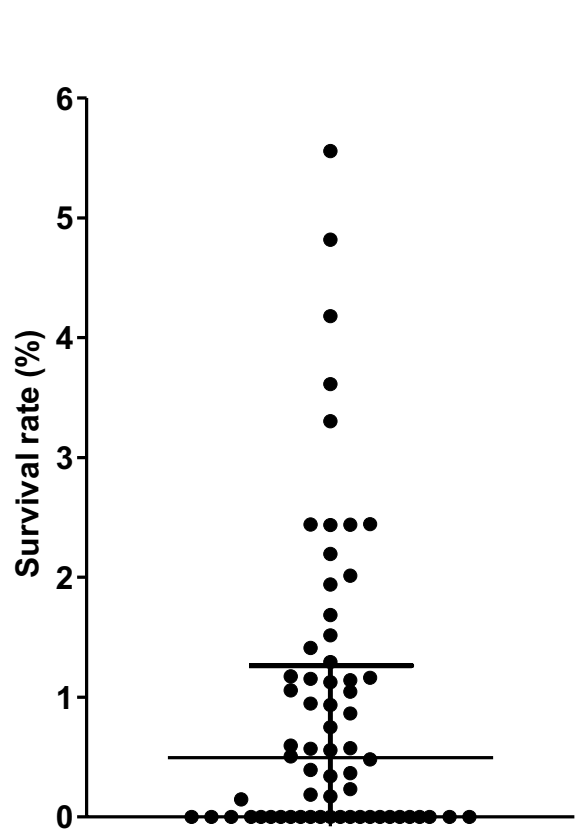

a

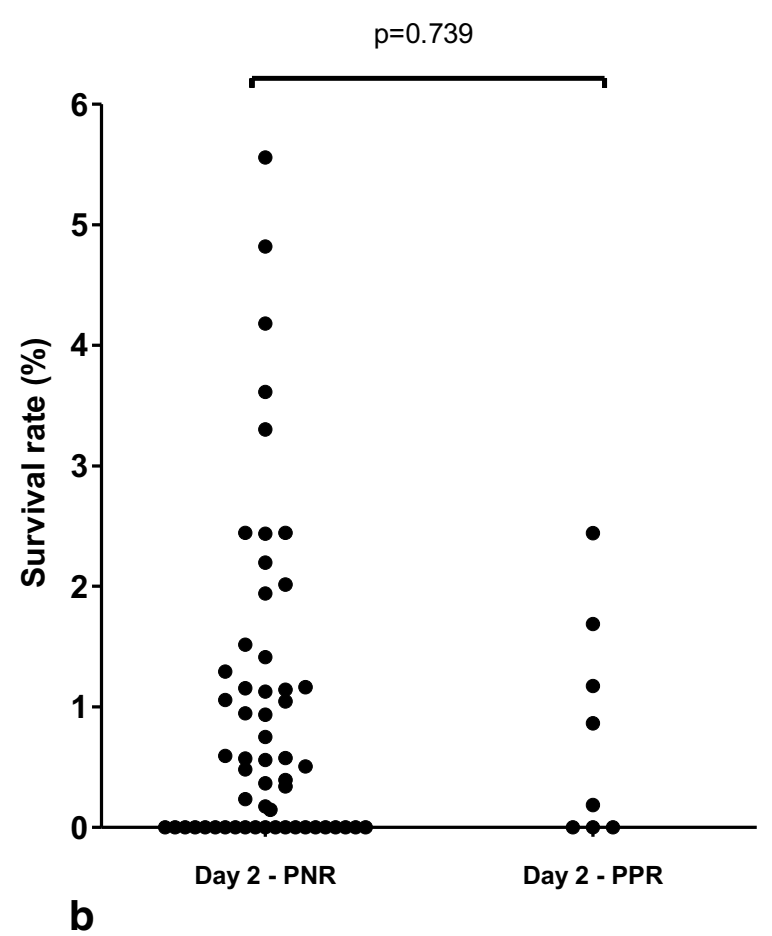

Fig. 2 Ex-vivo survival after exposure to DHA. Ex-vivo RSA exposed 93 isolates obtained from patients with uncomplicated falciparum malaria to $700 \mathrm{nM}$ DHA or dimethyl sulfoxide (DHA solvent) during $6 \mathrm{~h}$. Data were considered interpretable if the growth rate was $\geq 1$. a Results obtained in the ex vivo RSA from the 64 isolates with interpretable data (growth rate was $\geq 1$ ) are expressed as the survival rate of $P$. falciparum parasites after a 6 hour exposure to $700 \mathrm{nM}$ DHA compared with dimethyl sulfoxide (DHA solvent). The horizontal line represents the mean and whiskers the interquartile range. $\mathbf{b}$ Survival rates obtained in the ex vivo RSA from the 64 isolates with interpretable data are expressed in the two groups, day 2 PNR and day 2 PPR. Day 2 PNR represented the proportion of non-parasitaemic patients on day 2 whereas day PPR 2 represented the proportion of patients remaining parasitaemic on day 2, respectively. The ex vivo RSA data were analysed using the $t$-test for two group comparisons

by the variability in parasite clearance time as no significant difference in survival rates was observed between PPR and PNR isolates. Moreover, the four isolates with a survival rate higher than the mean plus 2 standard deviations showed no remaining parasitaemia on day 2 and had no $k 13$ polymorphism. These results are comparable to those observed by Ashley et al. who occasionally found patients with parasite clearance half-life values more than 5 hours but without association with $k 13$ polymorphisms [21]. These data were consistent with the survival rates obtained in the only ex vivo RSA study performed in East Africa (Uganda), which varied between 0.7 and 1.9\% [17]. However, in their study, Cooper et al. reported only three out of 43 DHA-pulsed cultures with healthy-appearing parasites [17], which is lower than that obtained in the present work (40/64). Other factors unrelated to ART resistance could influence the dispersion in survival rate and/or a low number of positive DHA-pulsed cultures, such as a lower growth rate during the ex vivo RSA or a difference in parasite age or density at the initiation of the test. Indeed Witkowski et al. pointed out in Cambodia that a growth rate higher than 1 is required to correctly analyse survival rate from ex vivo RSA [15]. Witkowski et al. also showed that RSA performed with late-ring or trophozoite forms does no longer identify a slow-clearance infection, by contrast to RSA with early-ring forms [15]. So storage and transport conditions of parasite isolates before processing are highly important in order to have the earliest parasite stages and then to obtain the more reliable survival rates in the ex vivo RSA.

In this study, the genotypic and ex vivo phenotypic profiles were associated with a rapid parasite reduction ratio. At day 2, 87.5\% (56/64) of patients had no detectable parasitaemia, the remaining $12.5 \%$ had a low P. falciparum density and the prevalence of clinical symptoms was highly reduced for all patients. Simple measure of parasitaemia is a good approximation of the parasite clearance rate [21]. In this study, the clinical and parasitological assessment was chosen to be based on a follow-up with two time points, at enrolment (day 0) and at day 2, regarding the fast parasite clearance time observed in Africa [23-25] as in the 
Table 2 Clinical and parasitological characteristics at day 2

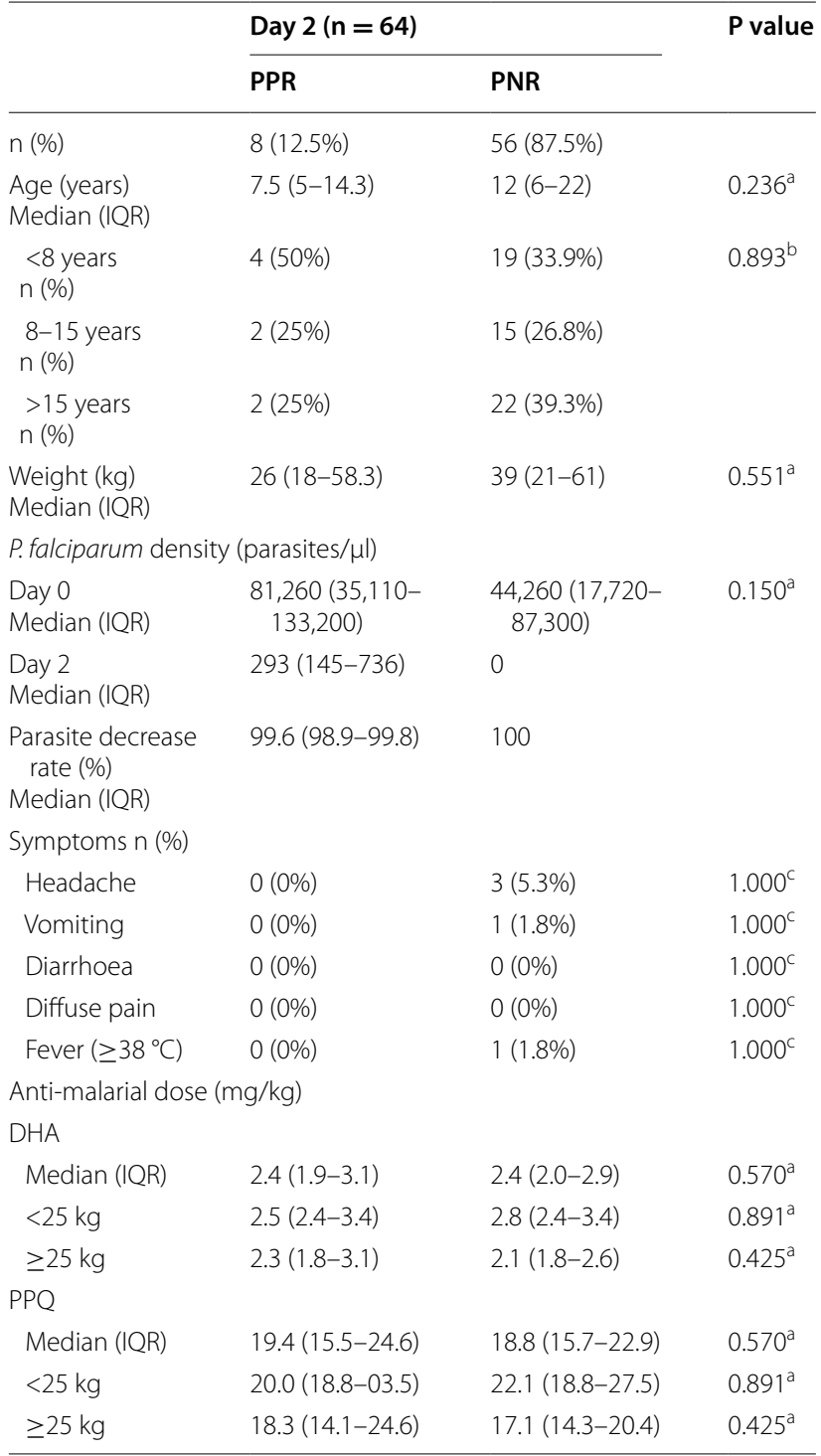

Day 2 PPR: proportion of patients remaining parasitaemic on day 2

Day 2 PNR: proportion of patients without parasitaemia on day 2

a Mann-Whitney $U$ test

${ }^{b} \mathrm{Chi}^{2}$ test

c Fisher's exact test

Nkol-Eton healthcare centre. Despite a possible correlation between parasitaemia at day $2 / 3$ and at enrolment [26], the assessment of the proportion of patients with a detectable parasitaemia at day 2 seemed a relevant parameter for quantitatively comparing with genotypic and ex vivo phenotypic profiles, and for rapidly detecting any change in P. falciparum susceptibility. Moreover this follow-up is easier to implement in healthcare centres compared to the six measurements within the first $48 \mathrm{~h}$ of treatment that require hospitalization of patients and a dedicated staff.
This study finally shows the feasibility of an overall, effective and inexpensive assessment of the susceptibility of $P$. falciparum to artemisinin derivatives that could be implemented in most endemic countries with minimal equipment (listed in "Methods" section, with the exception of sequencing that can be achieved at a later stage) but with experienced and well-trained technicians for the correct execution of the RSA.

\section{Conclusions}

This study gives reassurance about the level of ART resistance in Cameroon and provides baseline data of $k 13$ polymorphism, survival rate in ex vivo RSA and PPR profiles that will be essential for monitoring ART-derivative susceptibility in Africa. Similar studies will be required in other African countries to obtain an exhaustive view of these phenotypic data.

\section{Abbreviations}

ACD: acid-citrate-dextrose; ACT: artemisinin-based combination therapy; ART: artemisinin; DHA: dihydroartemisinin; DNA: deoxyribonucleic acid; DMSO: dimethyl sulfoxide; ETF: early treatment failure; IC50: 50\% inhibitory concentration; IC95: 95\% confidence interval; IQR: interquartile range; PCR: polymerase reaction chain; PNR: parasite negative rate; PPR: parasite positive rate; PPQ: piperaquine; RSA: ring-stage survival assay; SNP: single nucleotide polymorphism; WHO: World Health Organization.

\section{Authors' contributions}

SM, IM and AB conceived and designed the study. CNM and FAAM collected samples and information from participants. SM, JNT performed ex vivo RSA and SEN, LA and MTT performed microscopic examination. SM and JNT extracted DNA from blood and carried out all molecular methods. XI performed the statistical analysis. SM, IM and AB drafted the manuscript. PHAA participated in coordination. All authors read and approved the final manuscript.

\section{Author details}

${ }^{1}$ Centre de Physiopathologie de Toulouse Purpan, INSERM U1043, CNRS UMR5282, Université de Toulouse, Toulouse, France. ${ }^{2}$ Laboratoire d'Entomologie Médicale, Organisation de Coordination pour la lutte contre les Endémies en Afrique Centrale, Yaoundé, Cameroon. ${ }^{3}$ Faculté de Médecine et des Sciences Pharmaceutiques, Université de Douala, Douala, Cameroon. ${ }^{4}$ Service de Parasitologie-Mycologie, CHU Toulouse, Toulouse, France. ${ }^{5}$ UMR MIVEGEC, IRD 224-CNRS5290-UM, Institut de Recherche pour le développement, Montpellier, France. ${ }^{6}$ Dispensaire de Nkol-Eton, BP894 Yaounde, Cameroon.

\section{Acknowledgements}

The authors thank the patients and health staff of the Nkol-Eton health care centre in Yaounde for their participation in the study. We are grateful to the Genome and Transcriptome core facilities in Centre Hospitalier Universtaire de Purpan for sequencing.

\section{Competing interests}

The authors declare that they have no competing interests.

\section{Ethics approval and consent to participate}

All procedures involving human subjects used in this study were approved by the Cameroonian National Ethical Committee (statement n': 2015/04/582/CE/ CNERSH/SP). All patients were enrolled as volunteers after they or their parents had signed an informed consent form.

\section{Funding}

This study was supported in part by the French Agence Nationale de la Recherche (Grant ANR-13-BSV3-0018-0), by Institut National de la Santé et 
de la Recherche Médicale, by ParaFrap Consortium, and by core funding from the Institut de Recherche pour le Développement. JNT was supported by a scholarship from the Agence Universitaire de la Francophonie (AUF).

Received: 1 June 2016 Accepted: 17 November 2016

Published online: 26 November 2016

\section{References}

1. WHO: Briefing on malaria treatment guidelines and artemisinin monotherapies. Geneva:World Health Organization; 2006. http://www.who.int/ malaria/publications/atoz/meeting_briefing19april.pdf. Accessed Apr 2006.

2. Noedl H, Se Y, Schaecher K, Smith BL, Socheat D, Fukuda MM, et al. Evidence of artemisinin-resistant malaria in western Cambodia. N Engl J Med. 2008;359:2619-20.

3. Dondorp AM, Nosten F, Yi P, Das D, Phyo AP, Tarning J, et al. Artemisinin resistance in Plasmodium falciparum malaria. $N$ Engl J Med. 2009;361:455-67.

4. Phyo AP, Nkhoma S, Stepniewska K, Ashley EA, Nair S, McGready R, et al. Emergence of artemisinin-resistant malaria on the western border of Thailand: a longitudinal study. Lancet. 2012;379:1960-6.

5. Amaratunga C, Sreng S, Suon S, Phelps ES, Stepniewska K, Lim P, et al. Artemisinin-resistant Plasmodium falciparum in Pursat province, western Cambodia: a parasite clearance rate study. Lancet Infect Dis. 2012:12:851-8.

6. Hien TT, Thuy-Nhien NT, Phu NH, Boni MF, Thanh NV, Nha-Ca NT, et al. In vivo susceptibility of Plasmodium falciparum to artesunate in Binh Phuoc Province, Vietnam. Malar J. 2012;11:355.

7. Kyaw MP, Nyunt MH, Chit K, Aye MM, Aye KH, Aye MM, et al. Reduced susceptibility of Plasmodium falciparum to artesunate in southern Myanmar. PLOS ONE. 2013;8:e57689.

8. Snow RW, Trape JF, Marsh K. The past, present and future of childhood malaria mortality in Africa. Trends Parasitol. 2001;17:593-7.

9. Trape JF, Pison G, Preziosi MP, Enel C, Desgrees du Lou A, Delaunay V, et al. Impact of chloroquine resistance on malaria mortality. C R Acad Sci III. 1998:321:689-97.

10. Vreden SG, Jitan JK, Bansie RD, Adhin MR. Evidence of an increased incidence of day 3 parasitaemia in Suriname: an indicator of the emerging resistance of Plasmodium falciparum to artemether. Mem Inst Oswaldo Cruz. 2013;108:968-73.

11. Witkowski B, Lelievre J, Barragan MJ, Laurent V, Su XZ, Berry A, et al. Increased tolerance to artemisinin in Plasmodium falciparum is mediated by a quiescence mechanism. Antimicrob Agents Chemother. 2010;54:1872-7.

12. Menard S, Ben Haddou T, Ramadani AP, Ariey F, Iriart X, Beghain J, et al. Induction of multidrug tolerance in Plasmodium falciparum by extended artemisinin pressure. Emerg Infect Dis. 2015;21:1733-41.

13. Ariey F, Witkowski B, Amaratunga C, Beghain J, Langlois AC, Khim N, et al. A molecular marker of artemisinin-resistant Plasmodium falciparum malaria. Nature. 2014;505:50-5.
14. Straimer J, Gnadig NF, Witkowski B, Amaratunga C, Duru V, Ramadani AP, et al. Drug resistance. K13-propeller mutations confer artemisinin resistance in Plasmodium falciparum clinical isolates. Science. 2015;347:428-31.

15. Witkowski B, Amaratunga C, Khim N, Sreng S, Chim P, Kim S, et al. Novel phenotypic assays for the detection of artemisinin-resistant Plasmodium falciparum malaria in Cambodia: in vitro and ex vivo drug-response studies. Lancet Infect Dis. 2013;13:1043-9.

16. WHO: Status report on artemisinin and ACT resistance. World Health Organisation. 2015. http://www.who.int/malaria/publications/atoz/ status-rep-artemisinin-act-resistance-sept2015.pdf. Accessed Sep 2015.

17. Cooper RA, Conrad MD, Watson QD, Huezo SJ, Ninsiima H, Tumwebaze P, et al. Lack of artemisinin resistance in Plasmodium falciparum in Uganda based on parasitological and molecular assays. Antimicrob Agents Chemother. 2015;59:5061-4.

18. Torrentino-Madamet M, Fall B, Benoit N, Camara C, Amalvict R, Fall M, et al. Limited polymorphisms in $\mathrm{k} 13$ gene in Plasmodium falciparum isolates from Dakar, Senegal in 2012-2013. Malar J. 2014;13:472.

19. Kamau E, Campino S, Amenga-Etego L, Drury E, Ishengoma D, Johnson $\mathrm{K}$, et al. K13-propeller polymorphisms in Plasmodium falciparum parasites from sub-Saharan Africa. J Infect Dis. 2015;211:1352-5.

20. Hawkes M, Conroy AL, Opoka RO, Namasopo S, Zhong K, Liles WC, John CC, Kain KC. Slow clearance of Plasmodium falciparum in severe pediatric malaria, Uganda, 2011-2013. Emerg Infect Dis. 2015;21:1237-9.

21. Ashley EA, Dhorda M, Fairhurst RM, Amaratunga C, Lim P, Suon S, et al. Spread of artemisinin resistance in Plasmodium falciparum malaria. N Engl J Med. 2014:371:411-23.

22. Taylor SM, Parobek CM, DeConti DK, Kayentao K, Coulibaly SO, Greenwood BM, et al. Absence of putative artemisinin resistance mutations among Plasmodium falciparum in Sub-Saharan Africa: a molecular epidemiologic study. J Infect Dis. 2015;211:680-8.

23. Ndour PA, Lopera-Mesa TM, Diakite SA, Chiang S, Mouri O, Roussel C, et al. Plasmodium falciparum clearance is rapid and pitting independent in immune Malian children treated with artesunate for malaria. J Infect Dis. 2015;211:290-7.

24. Lopera-Mesa TM, Doumbia S, Chiang S, Zeituni AE, Konate DS, Doumbouya $\mathrm{M}$, et al. Plasmodium falciparum clearance rates in response to artesunate in Malian children with malaria: effect of acquired immunity. J Infect Dis. 2013;207:1655-63.

25. Zwang J, Dorsey G, Martensson A, d'Alessandro U, Ndiaye JL, Karema C, et al. Plasmodium falciparum clearance in clinical studies of artesunateamodiaquine and comparator treatments in sub-Saharan Africa, 1999-2009. Malar J. 2014;13:114.

26. WWARN. Clinical determinants of early parasitological response to ACTs in African patients with uncomplicated falciparum malaria: a literature review and meta-analysis of individual patient data. BMC Med. 2015;13:212.

\section{Submit your next manuscript to BioMed Central and we will help you at every step:}

- We accept pre-submission inquiries

- Our selector tool helps you to find the most relevant journal

- We provide round the clock customer support

- Convenient online submission

- Thorough peer review

- Inclusion in PubMed and all major indexing services

- Maximum visibility for your research

Submit your manuscript at www.biomedcentral.com/submit 